BULLETIN Bulletin hispanique

HISPANIQUE Université Michel de Montaigne Bordeaux

119-1 | 2017

Autorité et pouvoir dans le théâtre du Siècle d'Or

\title{
La culture équestre
}

le personnage face au pouvoir et l'auctoritas comique du dramaturge dans quelques comedias de Lope de Vega

\section{Marie-Eugénie Kaufmant}

\section{(2) OpenEdition}

1 Journals

Édition électronique

URL : http://journals.openedition.org/bulletinhispanique/4867

DOI : 10.4000/bulletinhispanique.4867

ISBN : 979-10-300-0142-6

ISSN : $1775-3821$

Éditeur

Presses universitaires de Bordeaux

Édition imprimée

Date de publication : 15 juin 2017

Pagination : 203-216

ISBN : 979-10-300-0141-9

ISSN : 0007-4640

Référence électronique

Marie-Eugénie Kaufmant, « La culture équestre », Bulletin hispanique [En ligne], 119-1 | 2017, mis en

ligne le 15 juin 2020, consulté le 14 septembre 2020. URL : http://journals.openedition.org/

bulletinhispanique/4867 ; DOI : https://doi.org/10.4000/bulletinhispanique.4867

Tous droits réservés 


\title{
La culture équestre, le personnage face au pouvoir et l'auctoritas comique du dramaturge dans quelques comedias de Lope de Vega
}

\author{
Marie-EugÉnie Kaufmant \\ Université de Caen Normandie (ERLIS)
}

Le service que rend le cheval implique une reconnaissance officielle qui évoque celle revendiquée par le dramaturge auprès du Roi. Plus que comme le piédestal d'une potestas, le cheval politique est dramaturgiquement représenté par Lope comme médiateur de l'auctoritas, à la fois celle du roi et celle du dramaturge.

Mots-clés: Lope de Vega, cheval, culture équestre, laquais, curialisation, mobilité socio-dramatique.

El servicio cumplido por el caballo implica un reconocimiento oficial que evoca el que reivindica el dramaturgo ante el Rey. Más que como el pedestal de una potestas, el caballo político es dramatúrgicamente representado por Lope como medianero de la auctoritas, tanto del rey como del dramaturgo.

Palabras claves: Lope de Vega, caballo, cultura ecuestre, lacayo, gracioso, transformación cortesana de la sociedad, movilidad socio-dramática.

The service rendered by the horse implies an official recognition, which evokes the one claimed by the dramatist before the king. More than the pedestal of potestas, the political horse is dramaturgically portrayed by Lope as a mediator of the auctoritas, both the king's and the dramatist's.

Keywords: Lope de Vega, horce, equestrian culture, lacayo, curialisation, sociodramatic mobility. 
D lus que tout autre animal dans l'histoire de notre bestiaire symbolique, le cheval devient un véritable symbole sociétal de pouvoir et d'autorité. Dès la Renaissance curiale, il s'érige en animal politique, du fait qu'il exprime « la nature particulière de la relation de l'homme à l'animal " ${ }^{1}$ selon le rapport de force entre humanité et animalité qui caractérise la symbolisation animalière politique. Dans le contexte de l'art équestre naissant à partir de la Renaissance qui remplace l'exclusif rapport de force de l'équitation guerrière, le cavalier fait le cheval mais l'inverse est tout aussi vrai : le cheval fait le cavalier, ou plus exactement ce qui constitue le couple équestre c'est cette dynamique d'harmonieuse synergie qui donne à cet animal une place privilégiée dans la hiérarchie animale symbolique. Le couple équestre symbolise, de ce fait, dès cette époque, tant l'autorité du monarque ${ }^{2}$ que la question de la réciprocité de l'engagement politique du roi vis-à-vis des sujets à son service selon le système curial. La culture équestre acquiert une fonction de représentation des relations hiérarchisées de la société à écuyers ${ }^{3}$ qui s'instaure alors. Particulièrement en Espagne, dans le cadre de la création par Philippe II à partir de 1567 des écuries royales de Cordoue, le cheval de pure race espagnole est considéré comme un prodigieux instrument politique, culturel et scientifique, que le souverain entend maîtriser et sélectionner ${ }^{4}$. Il n'est pas étonnant dès lors que la comedia nueva, façonnée par Lope de Vega à l'époque de l'avènement du " pure race espagnole " dans les Ecuries Royales de Cordoue, fonctionne comme caisse de résonnance poétique ${ }^{5}$ de cet essor " politique " au sens large de la culture équestre.

Dans la tradition des analyses de Bourdieu sur la création d'une sphère d'autonomie du champ intellectuel, Daniel Roche considère que la véritable culture équestre qui s'instaure en Europe à partir du $\mathrm{XVI}^{\mathrm{e}}$ siècle s'inscrit encore et tout particulièrement "dans la sphère de dépendance socio-politique " ${ }^{6}$. Or, les notions imbriquées d'autorité et de pouvoir prennent encore davantage d'épaisseur dès lors que l'on s'intéresse au champ intellectuel constitué par la production pionnière de comedias pour un théâtre commercial qui place le dramaturge au cour d'un marché et lui octroie une certaine indépendance économique et professionnelle. Plus encore que pour le reste des lettres

1. «Présentation : pour une analyse comparée des usages politiques de l'animal », dans Éric Baratay et als. (dir.), L'animal politique, Paris, l'Harmattan, 2003, p. 11-14 (la cit., p.12).

2. Voir l'analyse du couple équestre dans l'emblématique par Jean-Pierre Étienvre, "Introduction : pour une Histoire de la métaphore ", Littérature et politique en Espagne aux Siècles d'Or, Paris, Klincksieck, 1998, p. 11-27.

3. Pour la définition de ce concept de société à écuyers, voir Jean-Pierre Digard, Une histoire du cheval: art, techniques, société, Paris, Actes Sud, 2007, p. 141.

4. Voir Juan Carlos Altamirano, Historia y origen del caballo español: las caballerizas reales de Córdoba (1567-1800), Málaga, édition à compte d'auteur, 1998.

5. Un ouvrage issu de mon inédit d'HDR est en cours d'élaboration sur les fondements idéologiques et les mécanismes esthétiques de la poétique du cheval dans la comedia de Lope à Calderón.

6. Daniel Roche, Histoire de la culture équestre (XVI- $X I X^{e}$ siècle), III : connaissance et passion, Paris, Fayard, 2015, p. 56. 
espagnoles de l'époque, où la quête de la renommée auctoriale concerne tous les écrivains ${ }^{7}$, l'avènement de ce théâtre favorise une redéfinition de l'auctoritas, traditionnellement soumise aux modèles antiques et au pouvoir, et l'émergence conflictuelle d'une conscience auctoriale : le poète-dramaturge se trouve dans une position contradictoire entre les impositions mercantiles de sa professionnalisation lucrative et les anciennes nécessités clientélistes du mécénat ${ }^{8}$. Ce marché théâtral place le dramaturge entre la position de l'auctor créateur d'une œuvre, mais nécessitant, selon la tradition, l'appui d'une autorité, et la position de l'artifex, exécutant artisanal d'un ouvrage de composition de pièces de théâtre à succès populaire, dont le véritable maître (qui a autorité sur le texte) est le bien-nommé autor ou chef de troupe? ${ }^{9}$ Lope de Vega, qui, toute sa vie, lutta pour que soit reconnue son auctoritas dramaturgique, exprime donc logiquement, dans son œuvre comique et particulièrement à travers certains de ses personnages de comedias, certaines des inquiétudes du dramaturge. Il s'agit alors de démontrer, dans cet article, les rapports qu'entretient la poétique du cheval, telle que la conçoit Lope de Vega dans ses œuvres comme reflet esthétisé et idéologisé d'une culture équestre dominante dans la société du Siècle d'Or, avec ces velléités nobiliaires d'un dramaturge à la frontière entre mécénat et professionnalisation, à la conquête de l'autorité émanant de la reconnaissance de son œuvre de la part du pouvoir. Nous allons donc analyser selon quels fondements idéologiques, retravaillés dans le creuset poétique, ce symbole des relations curiales mais aussi de l'inspiration poétique qu'est le cheval, abondamment représenté dans la comedia lopesque, entre répétitivement dans la légitimation de l'autorité de personnages en quête de promotion et de reconnaissance de la part des sphères du pouvoir : le chevalier envisagé depuis la modernité curiale et le laquais gracioso. Le creuset poétique de la comedia sera appréhendé à travers le motif du cheval, symbole curial et politique mais aussi emblème d'une inventivité poétique jaillissant par le biais du référent mythique de Pégase, monture des Poètes, qui fait jaillir sous son sabot la source Hippocrène, lieu d'inspiration des Muses.

7. Pour l'analyse d'une autorité littéraire « définitoire » de la conscience auctoriale, voir l'étude de Karine Durin, "Autoridades controvertidas : erudición y heterodoxia en la España del siglo XVII ", dans Jesús M. Usunáriz, Edwin Williamson (eds.), La autoridad politica y el poder de las letras en el Siglo de Oro, Frankfurt/ Madrid, Vervuert/Iberoamericana, 2013, p. $43-57$ (pour cet aspect, p. 44-45).

8. Alejandro Garcia Reidy, Las musas rameras : oficio dramático y conciencia profesional en Lope de Vega, Madrid, Frankfort, Vervuert/Iberoamericana, 2013.

9. Voir l'émergence de cette conscience auctoriale à travers les prologues de Lope aux éditions de ses pièces, notamment dans le prólogo dialogístico, Parte XIX y la mejor parte de las comedias de Lope de Vega Carpio (Madrid, Juan González, 1624), D. Noguera et M. E. Kaufmant (ed.), site IdT, 2015, URL : http://www.idt.paris-sorbonne.fr. 
La comedia lopesque sédimente les temporalités historiques en s'intéressant préférentiellement et nostalgiquement à l'Histoire épique de l'Espagne mais en la réactualisant par un "effet de contemporanéité et de simultanéité " ${ }^{10}$ caractéristique des structures poétiques mises en œuvre au prisme de la fantaisie inventive du dramaturge. L'intérêt pour le Moyen Âge et la chevalerie en tant qu'objets de questionnement depuis la modernité alimente une réinvention constante de l'Histoire intégrée dans l'œuvre dramatique comme objet vivant de la part des dramaturges de l'époque des débuts du règne de Philippe III. L'anachronisme est constitutif de leur vision historique. En tant que porteuse d'une Histoire épique et moderne, la culture équestre intègre les questionnements idéologiques du Siècle d'Or dans le courant de la "vision temporelle totalisatrice $»^{11}$ d'une Histoire réinventée au sein de l'imaginaire dramatique sous l'éclairage de la modernité. La dislocation du monde féodal dans le contexte de la centralisation du pouvoir dans la société monarchoseigneuriale est un des thèmes constitutifs du théâtre de Lope ${ }^{12}$. À ce titre, Lope de Vega met en scène des chevaliers qui en appellent au pouvoir royal. Dès la fin du Moyen Âge, les monarques successifs ont tenté de centraliser le pouvoir et de mettre les chevaliers sous leur autorité directe par la notion de service cavalier qui s'instaure alors, créant ainsi un nouveau type de chevalerie dite "populaire » ou "villana». Le cheval, par le service qu'il permet, devient l'instrument d'un prestige chevaleresque, ouvrant l'accès à une certaine élite urbaine vers la fin du Moyen Âge, dont certains, notamment les chevaliers de sang, ne reconnaissent pas la légitimité. L'histoire complexe de la chevalerie médiévale fait du cheval le moteur d'un questionnement autour de l'autorité et du pouvoir social, notamment par le biais de cette "plébéisation de l'institution chevaleresque $»^{13}$. Les dramaturges des débuts du XVII' siècle que sont Vélez de Guevara, Guillén de Castro et Lope de Vega envisagent tous cette symbolisation chevaleresque au prisme des différentes significations déformées par la modernité qu'ils lui font prendre aux côtés du chevalier. La rime caballo/ vasallo vient souvent valider la symbolique d'un cheval qui dit au niveau textuel la loyauté d'un chevalier-vassal. Le cheval du chevalier exprime surtout la relation vertueuse du chevalier avec le pouvoir centralisateur des monarques.

10. Expression traduite empruntée à Nadine Ly, "Tiempo e historia en la Comedia : una poética de la contemporaneidad y simultaneidad ", dans Isabelle Rouane Soupault et Philippe Meunier (dir.), Tiempo e historia en el teatro del Siglo de Oro, Aix-en-Provence, Presses universitaires de Provence, 2015, p. 13-35 (la cit., p. 14).

11. Marc Vitse, Éléments pour une théorie du théatre espagnol du XVII siècle, Toulouse, Presses Universitaires du Mirail, 1990, p. 359.

12. Ibid., p. 352.

13. Termes traduits de Antonio Pérez Martín, «El estatuto jurídico de la caballería castellana ", dans Georges Martin (dir.), La chevalerie en Castille à la fin du Moyen Age : aspects sociaux, idéologiques et imaginaires, Paris, Ellipses, 2001, p. 13-26 (la cit., p. 17). 
Ainsi, par exemple, si le Cid médiéval est transformé par Guillén de Castro, dans Las mocedades del Cid et Las hazanas del Cid, en héros courtisan de la modernité au service de l'équilibre monarchique ${ }^{14}$, cet équilibre est notamment transcrit par le biais de l'image d'un parfait cavalier.

C'est aussi autour d'une notion d'équilibre que Lope de Vega structure la poétique de certains de ses personnages chevaliers dont le rapport au cheval est l'emblème d'une revendication récurrente de reconnaissance royale. L'équilibre est tout autre que chez Guillén de Castro car, chez Lope, le cheval ne fait pas le cavalier si le roi ne s'engage pas dans la relation de réciprocité que suppose le service chevaleresque. Lope inverse la notion de service cavalier en l'intégrant dans son système dramatique de réciprocité entre galants soumis à une poétique du miroir ${ }^{15}$ : le couple du galant et de son double royal reçoit l'influence du modèle d'autorité curiale où le cheval exprime les relations de dette politique engendrées par le service. Ainsi dans La resistencia honrada y condesa Matilde, le comte Jesualdo enfourche un cheval qui, dans un premier temps, est synonyme de la dette qu'il a, en tant que vassal, à l'égard de son souverain, comme le proclame la rime caballo/vasallo dans la réplique de son épouse Matilde, délaissée pour le combat : "Porque el freno / puesto tendrá el caballo, / id, que del Rey sois deudo y sois vasallo ${ }^{16}$. Le cheval est caractérisé par le frein du cheval harnaché au service du Prince, mais aussi implicitement symbole de la mesure au pouvoir. La même rime réapparaît lorsque Jesualdo est décrit revenant à pied aux côtés de son roi secouru au combat sur le cheval : "Al Rey saca del campo en su caballo / y viene a pie. ;Qué amigo, qué vasallo! ${ }^{17}$. Le prêt du cheval signe le pacte de réciprocité vassalique. C’est ainsi que la troisième occurrence de cette rime fait du cheval le symbole de la dette de loyauté lorsque le comte, agonisant suite au combat, fait don de son cheval au roi, espérant freiner les élans amoureux abusifs du roi en lui recommandant également sa future veuve Matilde, convoitée par ce même roi lascif: " aquel caballo / en que esta tarde os libré, / porque os acordéis que fue / su dueño vuestro vasallo ${ }^{18}$. Outre que le cheval apparaît comme une métaphore des relations galantes avec la dame, il est le médiateur d'une autorité respectueusement partagée entre le galant et son double royal, abusant potentiellement de son pouvoir par l'Eros que peut symboliser cette monture en l'absence de maitrise cavalière. Il résume les intérêts de pouvoir et d'Éros de relations socio-dramatiques qui s'éclairent par le modèle de transfert d'autorité que suppose le système curial à partir de la personne royale.

14. Voir Stefano Arata, "prologue ", Guillén de Castro, Las mocedades del Cid, Barcelone, Citica, 1996, p. LXVI-LXVIII.

15. Voir l'ouvrage de Maria Aranda, Le galant et son double, Toulouse, PUM, 1995.

16. Félix Lope de Vega Carpio, La resistencia honrada y condesa Matilde, Miguel M. GarcíaBermejo (ed.), dans Comedias de Lope de Vega. Parte II, vol. II, Lérida, Milenio, 1998, p. 699837 (la cit., v. 1449-1450).

17. Ibid., voir v. 2603-2604.

18. Ibid., v. 2804-2807. 
El servir con mala estrella offre un autre exemple de cheval significativement placé au cour d'un exemplum politique. Lope adapte à la scène la première nouvelle de la dixième journée du Décaméron. Mais, significativement, il remplace la mule de la nouvelle de Boccace par un cheval offert par le roi avec beaucoup de retard lorsque Rugero de Valois regagne la France après de bons services au souverain d'Espagne, découragé qu'il est de n'en recevoir aucune récompense. Dans une réplique du valet Turín dévoué au soin du cheval, la robe lustrée doit servir de faire-valoir du service accompli à l'égard du souverain dans une société qui voue un culte aux apparences : " en el pelo / se te luce que has servido $"{ }^{19}$. Cependant, comme la mule dans la nouvelle, le cheval devient l'acteur en chemin d'un geste scatologique hautement emblématique : il urine dans un ruisseau, ce qui le signale comme le symbole d'une attitude royale inappropriée à l'égard de sujets déjà nantis. Le cheval signale l'aberration d'un clientélisme royal injustifié par des récompenses destinées à des sujets qui ne le méritent pas. Dans cette pièce, Lope inverse le symbolisme du cheval comme service chevaleresque envers le suzerain pour en faire l'emblème animalisant de la reconnaissance royale aberrante et de relations de clientélisme curial malmenées qui ne confèrent pas l'autorité sur le principe du mérite. Le don du cheval au chevalier éclaire donc les aspirations du héros lopesque à la mobilité socio-dramatique dans un système curial où le cheval met en évidence la valorisation des transferts d'autorité entre le souverain et ses sujets.

Lope valorise donc le cheval du chevalier en contradiction avec la notion exclusive d'auxilium qu'il implique davantage au Moyen Âge. Son utilisation dramatique repose sur une sédimentation du symbolisme politique du cheval, qui, à l'époque moderne, devient image de la bonne gestion du monarque envers ses sujets. El primer Fajardo est une pièce intéressante à cet égard puisque Lope y met en scène une figure de chevalier populaire, tel qu'il pouvait s'en présenter dans l'Histoire de la Reconquête chrétienne. Juan, jeune chevalier en quête de gloire, fonde son lignage au nom du service chevaleresque par lequel il tue le Maure Abenalfajar, acte fondateur qui lui donne son nom Fajardo. Symboliquement, son cheval est présenté comme le descendant des juments maures, selon une poétique ambivalente de la pureté de sang d'un cheval ibérique largement métissé, qui est courante pour souligner l'ironie à l'égard de l'origine du lignage ou de la promotion des héros lopesques. La fin du premier acte de la pièce signale l'incohérente nécessité du héros : suite à un adoubement ruineux, il est obligé d'offrir son cheval à son soldat Pacheco pour qu'il le gage, afin de respecter l'apparence de libéralité dont toute personne élevée par le rang doit faire montre à l'égard de ses subalternes dans le système clientéliste qui est celui du Siècle d'Or. Le geste est d'autant plus décalé qu'historiquement, au Moyen Âge, il est répétitivement interdit aux chevaliers de gager leur cheval, qu'ils doivent entretenir en vue de la défense du royaume. La pauvreté du

19. Félix Lope de Vega Carpio, El servir con mala estrella, Laura Calvo Valdivielso (ed.), comedias de Lope de Vega. Parte VI, Vol. I, Lérida, Milenio, 2005, p. 649-770 (la cit. ,v. 22182219). 
chevalier apparait comme une aberration par rapport aux services qu'il rend au souverain par l'effort de guerre qu'il soutient :

\author{
FAJARDO \\ Quisiéralo [dinero] tener para prestallo, \\ ipor vida de Fajardo!, buen Pacheco; \\ mas si queréis, jugad sobre el caballo \\ que ayer me dio Garcijofre por trueco. \\ PACHECO \\ No tiene el Rey más principal vasallo. \\ ¿Qué estás tan pobre? \\ FAJARDO \\ Hame dejado en seco \\ este verano, como a humilde arroyo, \\ y el Conde, ausente, que era, en fin, mi apoyo; ${ }^{20}$
}

La rime caballo/vasallo vient de nouveau souligner la façon dont le cheval éclaire manifestement la quête d'autorité sociale d'un chevalier populaire que Lope intègre dans les nécessités de la société clientéliste : celle-ci repose sur la libéralité du souverain qui soutient le cercle vertueux des transferts d'autorité politique. Mais quelle est la motivation de cette poétique d'un personnage dont le service cavalier implique une reconnaissance, condamné qu'il est à la nécessité à défaut? Le discours anachronique de Fajardo, soumis au clientélisme aléatoire auprès du noble Garcijofre qui lui a offert le cheval, se rapproche des conditions de subsistance d'un dramaturge contraint aux aléas entrainés par le mécénat nobiliaire, notamment celui du duc de Sessa, à défaut de la reconnaissance royale. Lope élabore, de pièce en pièce, un tissu de références à la nécessité du chevalier en rapport avec le cheval. La trahison perpétrée du fait de l'aliénation du cheval est une prémonition de la trahison dont Fajardo, en mal de reconnaissance royale, va se rendre coupable, en passant dans le camp du roi Maure au troisième acte. Le ralliement à la cause du roi Maure entraîne aussitôt le don du propre cheval de ce dernier ${ }^{21}$. La libéralité du roi maure se trouve aux antipodes de la ladrerie du roi castillan. Dans sa lettre au roi, lue par le grand maitre, Fajardo reprend le motif équestre pour revendiquer la générosité reconnaissante du souverain, qui doit s'inscrire dans un cercle vertueux de conquête :

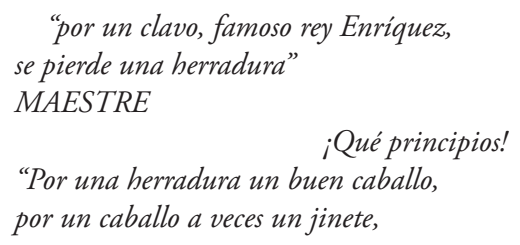

20. Félix Lope de Vega, El primer Fajardo, Jorge García López (ed.), Comedias de Lope de Vega. Parte VII, vol. II, Lérida, Milenio, 2008, p. 967-1087 (la cit., v. 823-830).

21. Ibid., voir v. 2397. 
L'originalité de Lope est de faire coöncider cet exemplum issu de la source lopesque, qui est la chronique de Francisco de Cascales $^{23}$, avec toute la dramatisation construite autour du cheval du chevalier : ce cheval, maillon du cercle vicieux de l'absence de reconnaissance royale, renvoie au cheval perdu au jeu avec tout son harnachement, jusqu'aux fers, au premier acte. La poétique du cheval dans El primer Fajardo sert à discuter l'autorité d'un roi qui n'est pas légitimée par une juste libéralité envers ses vassaux. Ainsi le don du cheval comme symbole de loyauté du chevalier s'inverse-t-il en exemplum de la gestion d'un pouvoir royal remis en cause dans son autorité qui demande la réciprocité vis-à-vis des services rendus.

Lope met en scène des chevaliers de rang moyen, proches du profil du caballero villano médiéval, comme Fajardo mais aussi comme Peribáńez dans Peribánez y el comendador de Ocaña. Le riche laboureur accède à une chevalerie anoblissante des mains d'instances locales sur la simple richesse de la possession d'une monture :

\author{
$Y$ en una yegua marchar, \\ notable para alcanzar \\ y famosa para huir, \\ si vieras como regía \\ Peribáñez sus soldados \\ te quitara mil cuidados. ${ }^{24}$
}

Si Peribáńez est promu chevalier populaire par le comendador, le chevauchement d'une jument et non du cheval, significateur d'un lignage noble par voie patrilinéaire, n'est pas bienséant pour un chevalier. Il justifie symboliquement que Peribánez soit la proie des exactions et abus du commandeur qui organise sa promotion pour mieux tenter de séduire son épouse Casilda en son absence. Peribánez est alors obligé de se rendre à la cour en chevauchant cette même jument à l'acte III pour recourir in fine à l'approbation du roi, après avoir assassiné le noble abuseur de son honneur. Comme Frondoso dans Fuenteovejuna, Péribáñez incarne, certes de façon ambiguë, "un anti-féodalisme au sein du féodalisme " ${ }^{25}$ et un ralliement à l'idéologie monarcho-seigneuriale du Siècle d'Or, mais il évoque aussi le conflit d'autorité qu'elle engendre. La jument, véhicule de la promotion sociale du

22. Ibid., v. 2859-2866.

23. Jorge García López, introduction à El primer Fajardo, Ibid., p. 972.

24. Lope de Vega, Peribáñez y el comendador de Ocaña, Juan María Marín (ed.), Madrid, Cátedra, 1997, III, v. 418-423.

25. Noël Salomon, Recherches sur le thème paysan dans la "comedia " au temps de Lope de Vega, Bordeaux, Institut d'Études Ibériques et Ibéro-américaines de l'Université de Bordeaux, 1965, voir p. 860-911 et, pour la formule citée, p. 889. 
chevalier populaire qu'est Peribánez, dénonce son manque de bienséance sans l'approbation royale. Femelle, elle signale le genre de prostitution chevaleresque dont il se rend coupable. Du reste, Casilda, l'épouse de Peribáńez, refuse-telle de chevaucher pour rejoindre la Cour royale à la fin de la pièce ${ }^{26}$. Entre autres symbolismes (notamment le rapport entre la poétique de la légèreté de la jument et l'honneur paysan), cette jument, "bestia destas de huir y alcanzar " ${ }^{27}$, bête de charge agricole transformée pour l'usage militaire, semble être destinée à la cavalerie légère qui s'est développée dans la tradition de la technique du harcèlement de l'équitation orientale. Elle rapproche Peribáńez de ces Maures de comedias morisques, juchés sur des juments fuyardes : elle intègre la fable de Peribáńez et le cheval de l'Histoire de la chevalerie populaire dans le tissu imaginaire qui disqualifie le héros tout autant qu'il l'insère dans une généalogie prestigieuse. La protection royale finale est le seul soutien du chevalier populaire. Le cheval, et tout particulièrement cette jument comme attribut de la figure ambiguë du paysan en armes qu'est Peribáńez, viennent une fois de plus éclairer la signification d'une conquête de l'autorité par un personnage en quête de l'approbation royale, qui peut créer un lignage ex-nibilo sans héritage patrilinéaire, comme l'exprime la monture « féminisée » du laboureur chevalier. Tout comme dans les pièces de privanza où l'action dramatique évoque le cheval comme un symbole de transfert d'autorité politique, la signification de la jument de Peribánez est bien politiquement ordonnée autour de la figuration d'une autorité qui demande à être confirmée par le roi. Sans doute est-ce également la signification de la jument d'un autre riche laboureur, Juan Labrador dans $E l$ villano en su rincón qui, lui, est caractérisé, dans une perspective apparemment inversée par rapport à Peribánéez, par sa relation fuyante à l'égard du pouvoir royal qui tente de faire respecter son autorité.

En tant que monture du chevalier médiéval ressaisi depuis la modernité curiale des comedias de privanza ou des drames paysans, le cheval lopesque pointe la volonté d'accession à l'autorité et surtout la question de la légitimité de sa valorisation.

\section{LE LAQUAIS DE L'ÉTRILLE AU SCEPTRE, EN QUÊTE D'AUCTORITAS COMIQUE}

Dans ce même contexte socio-dramatique, le personnage du valet bouffon semble délibérément fondé par Lope à partir de la figure d'un laquais dévoué au soin du cheval. Selon Jesús Gómez ${ }^{28}$, avant les années 1620 , le terme gracioso n'existe pas encore en tant que substantif dans sa signification de fonction dramatique. Dans les dramatis personae des pièces où Lope intègre ses premiers valets bouffons, c'est la terminologie lacayo qui désigne le personnage

26. Lope de Vega, Peribánez..., op. cit., III, voir v. 815-817.

27. Ibid., III, v. 529-530.

28. Jésús Gómez, "Una visión sobre el personaje del gracioso en la crítica actual ", $L a$ construcción de un personaje: el gracioso, Madrid, Fundamentos, 2005, p. 11-22. 
du gracioso. Le mot lacayo, d'introduction récente au XVI ${ }^{e}$ siècle, en rapport avec l'évolution curiale d'une culture équestre qui impose une domesticité spécifique, se pose alors comme une terminologie théâtrale équivalente aux fonctions dramatiques de galants et de dames. Il signale d'emblée le personnage dans la relation particulière qui l'unit à son maître : cette relation s'organise autour de la mobilité et de l'ouverture spatiale qui caractérise la comedia, puisque le laquais est le domestique qui précède à pied son maitre dans tous ses déplacements, particulièrement équestres comme le signale le Tesoro de Covarrubias $^{29}$. Le personnage comique revendique aussi perpétuellement son hyperfonctionnalité dramatique auprès d'un galant en quête d'ascension notamment dans les pièces palatines. Il place de ce fait, même de façon implicite, l'animal politique qu'est le cheval au cœur du système de mobilité socio-dramatique. À plusieurs reprises chez Lope, le laquais gracioso est mis en relation avec l'univers des écuries et du cheval jusqu'à assumer de façon peu réaliste la fonction de palefrenier. Mais, à l'inverse, la fonction dramatique du laquais se caractérise par l'ouverture et la mobilité au sein de l'univers palatin ou urbain, au point que sa polyfonctionnalité représente le comble de l'invraisemblance critiquée dans ce théâtre par les contemporains de la comedia. Le laquais est le représentant hypergénérique de la subalternité sociale, favorisée par la curialisation de la société concomitante de l'urbanisation qui est à l'origine de la perte d'indépendance de la figure comique selon la lecture sociologique et "spatiale» de ce personnage ${ }^{30}$. Mais pourquoi le laquais en particulier résume-t-il à lui seul l'ensemble des relations de subalternité de la société curiale symbolisée par une dramatis personae réduite dans ce théâtre ? Ricardo de Turia répond aux critiques d'invraisemblance de la figure dans son Apologético de las comedias españolas:

Y la introducción de los lacayos en las comedias no es porque entiendan que la persona de un lacayo sea para comunicalle negocios de estado y de gobierno, sino por no multiplicar interlocutores, porque si a cada principe le hubiesen de poner la casa que su estado pide, ni habría compañia, por numerosa que fuese, que bastase a representar la comedia, ni menos teatro (aunque fuese un coliseo) de bastante capacidad a tantas figuras, y asi hace el lacayo las de todos los criados de aquel principe... ${ }^{31}$

Le laquais apparaît donc comme la représentation stylisée et synthétique de la diversité des médiateurs de subordination qu'impose la curialisation de la société. Le laquais, plus que tout autre domestique, est associé à la mobilité équestre et ce qu'elle symbolise, l'écurie étant tournée vers l'extériorité distinctive, un faire-valoir du maitre en rapport avec une culture équestre qui

29. Covarrubias, Tesoro de la lengua castellana o española, Madrid, Castalia, 1994, entrée «lacayo".

30. Voir M. Vitse, "El imperio del gracioso: historia y espacio o del gracioso a lo gracioso ", Criticón, 60, 1994, p. 143-148.

31. Ricardo de Turia, "Apologético de las comedias españolas ", Norte de la poesía española, Préface, Alejandro García Reidy (ed.), Valencia, Felipe Mey, 1616, dans site IdT, supra, fol. A3v et A4r, URL. 
doit favoriser l'autorité et la mobilité sociale. Le métier de laquais apparait comme un premier pas vers le clientélisme. Ainsi les paysans frustes Bruno et Fileto, dans El villano en su rincón, expriment-ils ce qui fait la noblesse du nouveau statut qui leur est proposé : face au labeur et au travail mécanique de la terre, les deux futurs laquais apprécient ce moyen de pénétrer les cercles privilégiés du palais par l'écurie et un "Oficio honrado $\|^{32}$ que Lope semble burlesquement défendre comme un moyen d'ascension sociale au fil de ses pièces, même pour un laquais qui se fait invraisemblable palefrenier à ses heures. Le maniement de l'étrille pour lustrer la robe du cheval constitue pour le laquais lopesque un faire-valoir du service rendu au roi par son maître. Face au don du cheval par le roi à Rugero dans El servir con mala estrella, le valet Turín soigne la robe d'un animal qui devient un symbole du cercle vertueux du clientélisme récompensé et du transfert d'autorité qui retentit aux différents niveaux de l'échelle curiale.

Les laquais lopesques revendiquent couramment l'invraisemblance d'une polyfonctionnalité dramatique qui les conduit du maniement de l'étrille à la fréquentation des sceptres royaux pour le soin des cœurs et la promotion du galant aux amours royales: "¿Quién metió a mis almohazas / con los cetros de los reyes? "\$33, s'exclame Hernandillo, le laquais en quête de promotion royale dans La ocasión perdida. Si cette pièce met en scène l'occasion de promotion politique perdue par le galant comme par son valet entremetteur, la mobilité ascensionnelle des amours palatines du secrétaire Teodoro et de la comtesse Diana dans El perro del hortelano repose bien plus fructueusement sur l'inventivité du laquais Tristán. Pour celui-ci, la fonction du laquais comme représentation sociale de la noblesse du maitre est pleinement justifiée dès lors que la réalité première de sa fonction est d'aider son maître à se hisser sur le cheval social comme emblème du paraître : "Escalera del señor / si va a caballo, un discreto / nos llamó, pues a su cara / se sube por nuestros cuerpos " "34. Le corps $\mathrm{du}$ laquais comme corps politique qui sert de marche-pied au maître pour se hisser sur le cheval d'autorité éclaire la polyfonctionnalité de Tristán dans l'intrigue palatine. Le laquais est celui qui met le pied du galant à l'étrier de l'ascenseur social symbolique qu'est le cheval dans la société curiale.

La culture équestre se trouve donc placée par Lope au centre d'une comedia dont le système socio-dramatique condense les tensions politiques d'ascension sociale de la société curiale du Siècle d'Or. Comme les chevaliers populaires en quête de valorisation de leur autorité par le roi, le laquais, par l'inventivité qu'il déploie dans la mobilité socio-dramatique et dont il attend une reconnaissance parfois directement adressée au souverain, devient une figure de l'auto-dérision d'un Lope qui espère la reconnaissance royale de ses services en tant que

32. Félix Lope de Vega Carpio, El villano en su rincón, Juan María Marín (ed.), Madrid, Cátedra, 1999, v. 2702.

33. Lope de Vega, La ocasión perdida, Enrico di Pastena (ed.), Comedias de Lope de Vega. Parte II, vol. I, Lérida, Milenio, 1998, p. 267-395, v. 2173-2174.

34. Lope de Vega, El perro del hortelano, Mauro Armiño (ed.), Madrid, Castalia, 1970, v. $612-615$. 
dramaturge. Ainsi, à titre d'exemple, sans une argumentation qui ne peut être davantage étayée par l'analyse littérale dans le cadre de cette publication, dans La humildad y la soberbia, la figure du laquais Lope, qui quémande un poste de chroniqueur auprès du roi afin de sortir de sa condition laborieuse, représente la mise en abyme de la condition sociale du poète-client :

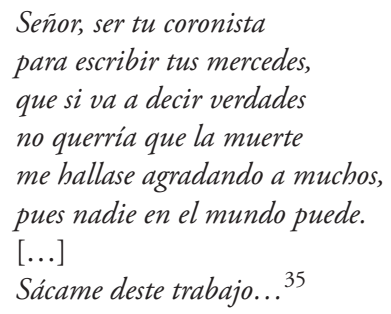

Comme le remarque déjà Noël Salomon pour cet extrait et d'autres références du laquais Lope dans la pièce ${ }^{36}$, la sollicitation ne peut être moins voilée à l'égard de Philippe III par un Lope qui ne prend pas même le soin d'utiliser un pseudonyme. Il prouve combien Lope, dans son auto-dérision, entrevoit dans le personnage du laquais, au service du cheval et, au-delà, de la représentation du maître, une image de son service en tant que dramaturge, un service qui mérite une récompense qui extirpe l'auteur de sa condition laborieuse. Cette identification est rendue possible par le symbolisme de promotion sociale que revêt la culture équestre et qui nourrit la relation que le laquais entretient avec le cheval, représentant, de façon burlesque, tout autant la promotion sociale que la noblesse et l'inventivité littéraires. Voilà comment le cheval s'immisce pleinement dans un théâtre qui tente de gagner ses lettres de noblesse sous l'étrille lustrante du lacayo de comedias auquel Lope s'identifie, intégrant avec cocasserie la critique gongoriste, à une époque où l'écriture dramatique pour le marché théâtral est entachée de la notion avilissante d'art mécanique et mercantile. A ce titre, le laquais Hernandillo, dans La ocasión perdida, revendique pleinement la noblesse de son statut et évoque les critiques faites à Lope en tant que lacayo de comedias par les gongoristes :

No hay en mi linaje ofensa, los envidiosos lo digan.

La almohaza es mi defensa, que los trabajos obligan a lo que el hombre no piensa. Mil, con rojos y amarillos hábitos, hacen corrillos

35. Lope de Vega, La humildad y la soberbia, Miguel Zugasti (ed.), Comedias de Lope de Vega. Parte X, I, Lérida, Milenio, 2010, p. 485-621, v. 2084-2089 et 2098.

36. Noël Salomon, "Sur quelques problèmes de sociologie théâtrale posés par La humildad y la soberbia, comedia de Lope de Vega ", dans Dramaturgie et Société, Rapports entre l'oeuvre théatrale, son interprétation et son public aux XVI et XVIF siècles, Jean Jacquot (éd.), I, Paris, CNRS, 1968, p. 13-30. 
contra el lacayo que ves, que puede honrar un pavés con diez y nueve castillos. ${ }^{37}$

La réplique pourrait bien être une réponse directe aux vers du sonnet attribué à Góngora dont l'auteur l'accusait de faire figurer en-tête de son Arcadia le blason aux dix-neuf tours de Bernardo del Carpio, qu'il revendique comme ancêtre. Dans ce sonnet, Lope est invité à revoir à la baisse ses velléités d'autorité nobiliaire en retournant à la réalité prosaïque de sa profession de dramaturge, qui est associée métaphoriquement au vil maniement de l'étrille pour débarrasser un Pégase, rabaissé au rang de roussin ailé, des larves des parasites : "Vuelva a su oficio, y al rocín alado / en el teatro sáquele los reznos " ${ }^{38}$. A l'inverse, l'étrille est utilisée, dans la bouche du laquais aux aspirations aristocratiques de La humildad y la soberbia, comme une référence à l'écriture dramatique et poétique qui lustre l'auctoritas nobiliaire du dramaturge mise à mal.

En vertu du symbolisme curial de la culture équestre, le cheval favorise le questionnement de la valorisation de l'auctoritas des personnages du chevalier populaire ou du laquais en mal de reconnaissance royale. La comedia d'un dramaturge professionnel, dont la fierté auctoriale continue de se justifier sur le principe de l'autorité en rapport avec le pouvoir, intègre cet animal politique, avec une nuance de dérision à partir des métaphores employées par la critique gongoriste. Par le biais du symbolisme mythique de Pégase, le cheval lustré par le laquais représente à la fois l'inventivité laborieuse et la noblesse littéraire d'une comedia qui doit retentir sur l'auctoritas du dramaturge et entrainer sa valorisation par le pouvoir royal. Le cheval permet de mettre en valeur des relations socio-dramatiques d'inspiration curiale où l'autorité du roi est mise en question par la délégation de son pouvoir suprême et l'exercice de sa faveur vis-à-vis des fonctions de serviteurs qui le soutiennent ${ }^{39}$. Pour Lope, le cheval n'apparaît pas tant comme symbole de potestas que comme médiateur d'auctoritas, une autorité politique qui repose sur la reconnaissance à l'égard du serviteur. Ce symbole curial d'un défi voilé face à l'autorité royale, utilisé par des personnages qui, par ailleurs, se soumettent au pouvoir par le service que suppose le cheval, est une façon détournée de mettre le pouvoir devant ses responsabilités, en lui "rappel[ant] les valeurs proclamées $"^{40}$. Si le théâtre

37. La ocasión perdida, op. cit., v. 2370-2379.

38. Luis de Góngora, Sonetos completos, Madrid, Castalia, 1992, A la "Arcadia" de Lope de Vega Carpio, p. 261.

39. Au sujet des intérêts complexes de la délégation d'autorité et de pouvoir auprès des conseillers du roi dans les œuvres théâtrales, voir Christoph Strosetzki, «Autoridad y poder del consejero en la comedia histórica del Siglo de Oro ", dans Autoridad política y el poder de las letras, op. cit. p. 59-72.

40. Expression traduite empruntée à Ignacio Arellano, Los rostros del poder en el Siglo de Oro : ingenio y espectáculo, Sevilla, Renacimiento, 2011, p. 311. 
classique peut se présenter à première vue -et simplement à première vue, précise Francisco Ruiz Ramón- comme un laquais au service de l'idéologie dominante ${ }^{41}$, ces serviteurs laborieux en rapport avec le cheval politique, sous l'apparence desquels se cache parfois le dramaturge, manient tantôt l'étrille, tantôt la brosse à reluire pour obtenir la faveur du Roi, garante d'autorité. 